\title{
Neuroprotective Activities of Sodium Valproate in a Murine Model of Human Immunodeficiency Virus-1 Encephalitis
}

\author{
Huanyu Dou, ${ }^{1,2}$ Kevin Birusingh, ${ }^{1,2}$ Jill Faraci, ${ }^{1,2}$ Santhi Gorantla, ${ }^{1,2}$ Larisa Y. Poluektova, ${ }^{1,2}$ Sanjay B. Maggirwar, ${ }^{5}$ \\ Stephen Dewhurst, ${ }^{5}$ Harris A. Gelbard, ${ }^{4,5}$ and Howard E. Gendelman ${ }^{1,2,3}$ \\ ${ }^{1}$ Center for Neurovirology and Neurodegenerative Disorders, ${ }^{2}$ Department of Pathology and Microbiology, and ${ }^{3}$ Department of Internal Medicine, \\ University of Nebraska Medical Center, Omaha, Nebraska 68198-5215, ${ }^{4}$ Center for Aging and Developmental Biology, and ${ }^{5}$ Department of Neurology, \\ Pediatrics, and Microbiology and Immunology, University of Rochester Medical Center, Rochester, New York 14642
}

Human immunodeficiency virus-1 (HIV-1) infection of the nervous system can result in neuroinflammatory events leading first to neuronal dysfunction then to cognitive and behavioral impairments in infected people. The multifaceted nature of the disease process, commonly called HIV-1-associated dementia (HAD), provides a number of adjunctive therapeutic opportunities. One proposed adjunctive therapy is sodium valproate (VPA), an anticonvulsant known to promote neurite outgrowth and increase $\beta$-catenin through inhibiting glycogen synthase kinase $3 \beta$ activity and tau phosphorylation. We now show that VPA treatment of rat cortical neurons exposed to HIV-1 gp120 prevents resultant neurotoxic activities. This includes the induction of significant neurite outgrowth and microtubuleassociated protein 2 (MAP-2) and neuron-specific nuclear protein (NeuN) antigens in affected neuronal cell bodies and processes. Similarly, VPA protects severe combined immunodeficient (SCID) mice against the neurodegeneration of $\mathrm{HIV}-1_{\mathrm{ADA}}$ infected monocytederived macrophages (MDMs). In SCID mice with HIV-1 MDM-induced encephalitis, VPA treatment significantly reduced neuronal phosphorylated $\beta$-catenin and tau without affecting HIV-1 replication or glial activation. We conclude that VPA protects neurons against HIV-1 infected MDM neurotoxicity, possibly through its effects on the phosphorylation of tau and $\beta$-catenin. The use of VPA as an adjuvant in treatment of human HAD is being pursued.

Key words: HIV-1 associated dementia; HIV-1 encephalitis; monocyte-derived macrophages; sodium valproate; neuroprotection; severe combined immunodeficient mice

\section{Introduction}

Progressive human immunodeficiency virus type one (HIV-1) infection leads to immune suppression and to a constellation of CNS disorders (Gendelman et al., 2003). In its most severe form, cognitive, motor, and behavior abnormalities predominate and are termed HIV-1-associated dementia (HAD). Although the incidence of HAD has been reduced to $<10 \%$ after the use of potent combination anti-retroviral therapy (PCAT), disease prevalence has remained constant (Sacktor et al., 2001). The pathological hallmark of HAD is HIV-1 encephalitis (HIVE). HIVE is characterized by the accumulation of virus-infected multinucleated giant cells in white and deep gray matter, along with myelin pallor and astrocytosis. Dendritic pruning and neuronal vacuolation are morphological correlates of neuronal damage (Masliah et al., 1992a). A unique feature of HIVE is that the principal viral target cell in the brain is not the neuron but the mononuclear phagocyte (MP; microglia and perivascular macrophages) (for review, see

Received July 9, 2003; revised Aug. 8, 2003; accepted Aug. 22, 2003.

This work was supported by National Institute of Neurological Disorders and Stroke Grants R37 NS36126 and R01NS034239 (H.E.G.) and National Institutes of Mental Health Grants P01MH64570 (H.A.G., H.E.G.) and R01 MH56838 (H.A.G.). We thank Robin Taylor for outstanding administrative and computer support, Janice Taylor for help with confocal microscopy, and Dr. Jenae Limoges for critical reading and editing of this manuscript.

Correspondence should be addressed to Dr. Howard E. Gendelman, Center for Neurovirology and Neurodegenerative Disorders, University of Nebraska Medical Center, Omaha, NE 68198-5215. E-mail: hegendel@unmc.edu. Copyright $\odot 2003$ Society for Neuroscience $\quad 0270-6474 / 03 / 239162-09 \$ 15.00 / 0$
Lipton and Gendelman, 1995). Disease occurs as a consequence of the secretion of viral and cellular neurotoxins by activated and virus-infected MP (Genis et al., 1992; Anderson et al., 2002).

In recent years significant attention was focused on the development of adjunctive therapies for HAD (Schifitto et al., 2001; Stern et al., 2001) for several reasons. First, PCAT has restricted efficacy in treatment of HAD because of limited CNS antiretroviral penetrance, potential for virus-back mutation and the development of HIV resistant phenotypes (Kravcik et al., 1999; Ledergerber et al., 1999; Swindells et al., 1999). Second, the neuropathogenesis of HIV-1 infection lends itself to multifaceted therapeutic approaches. MP infection elicits a cascade of paracrine immune events that lead to neuronal death (Gendelman et al., 2003). Third, only a small proportion of infected people have access to PCAT. Fourth, overlapping pathogenic mechanisms are now known to be operative between HAD and other neurodegenerative disorders (for example, Alzheimer's and Parkinson's diseases) (Gendelman, 2002). Such drugs would have broad applicability and could include neurotrophins, anti-inflammatory, anti-oxidants, and other compounds that block pathways for neuronal demise (Schifitto et al., 2001).

One drug that may be considered an adjuvant for HAD is sodium valproate (VPA). VPA promotes neurite outgrowth (Illig et al., 2000; O'Leary et al., 2000), increases synapsin I clustering, activates extracellular signal-regulated kinases, increases growth 
cone size and its associated protein 43 and bcl-2, and affects nerve regeneration (Manji et al., 2000; Hall et al., 2002; Tariot et al., 2002). Through its abilities to inhibit glycogen synthase kinase-3 $\beta$ (GSK-3 $\beta$ ) (Chen et al., 1999), VPA may play a role in blocking the synthesis of pro-apoptotic factors that contribute to neuronal loss (Perez et al., 2003). In this regard, significant neuroprotective effects of VPA in both laboratory and animal model systems of human HIVE (Persidsky et al., 1995; Zheng et al., 2001) were demonstrated. The neuroprotectant effects of VPA were shown to be independent of the levels of virus or the numbers of immune competent monocyte-derived macrophages (MDMs), but paralleled GSK-3 $\beta$ inhibition.

\section{Materials and Methods}

Primary human monocyte isolation and HIV-1 infection. Monocytes were obtained from leukophoresis of HIV-1, 2, and hepatitis B seronegative donors and purified by countercurrent centrifugal elutriation. Cells were cultured with $10 \%$ heat-inactivated pooled human serum, 1\% glutamine (Sigma, St. Louis, MO), $10 \mu \mathrm{g} / \mathrm{ml}$ ciprofloxacin (Sigma), and $1000 \mathrm{U} / \mathrm{ml}$ highly purified recombinant human macrophage colony stimulating factor (MCSF) (a generous gift from Genetics Institute, Inc., Cambridge, MA). After $7 \mathrm{~d}$ the MDM were infected with HIV-1 $1_{\mathrm{ADA}}$ (a macrophage tropic viral strain) at multiplicity of infection of 0.01 (Gendelman et al., 1988).

Rat cortical neurons. Cerebral cultures, containing neurons and glia in similar proportions to that found in the brain, were derived from the cerebral hemisphere of embryonic Sprague Dawley rats on day 17 of gestation and cultured as described previously (Yeh et al., 2000) after dissociation in $0.027 \%$ trypsin. Neuron-enriched cells were resuspended in neurobasal medium (Invitrogen, Grand Island, NY) with heatinactivated fetal calf serum supplemented with B-27, $500 \mu \mathrm{M}$ glutamine, and $25 \mu \mathrm{M}$ glutamate then seeded at a density of $2.8 \times 10^{5} \mathrm{cells} / \mathrm{cm}^{2}$ on polyethylenimine-coated 24 and 6 well plates. After $7 \mathrm{~d}$ of cultivation the cells were exposed to $5 \mathrm{~nm} \mathrm{HIV}-1_{\text {ADA }}$ gp120 and $3 \mathrm{~mm} \mathrm{VPA}$ for $5 \mathrm{~d}$. The HIV-1 $1_{\text {ADA }}$ gp120 used in these assays was purified by immunoaffinity chromatography to $>99.9 \%$ homogeneity (National Institutes of Health AIDS Research and Reference Reagent Program).

Severe combined immunodeficient mouse model of HIVE. Four-weekold male CB-17/IcrCrl-SCIDbr mice were purchased from Charles River Laboratory (Wilmington, WA). Animals were maintained in sterile microisolator cages under pathogen-free conditions in the Laboratory of Animal Medicine at the University of Nebraska Medical Center in accordance with ethical guidelines for care of laboratory animals set forth by the National Institutes of Health. HIV- $1_{\mathrm{ADA}}$-infected MDMs $\left(1.5 \times 10^{5}\right.$ cells in $5 \mu \mathrm{l}$ ) were stereotactically injected intracranially after $1 \mathrm{~d}$ of viral infection and referred to as HIVE mice (Persidsky and Gendelman, 2002). Replicate severe combined immunodeficient (SCID) mice received intracranial injections of media (sham-operated) and served as controls. Eleven animals were included in each group. One day after injection an additional group of 11 HIVE and 9 sham-operated mice were treated with VPA at $200 \mathrm{mg} \cdot \mathrm{kg}^{-1} \cdot \mathrm{d}^{-1}$. Animals were killed at 7 , 14 , and $21 \mathrm{~d}$ after MDM injections.

Histopathology and image analysis. Brain tissue was collected at necropsy, fixed in 4\% phosphate-buffered paraformaldehyde, and embedded in paraffin. Paraffin blocks were cut until the injection site of the human MDM was identified. HIV-1 p24 antigen (clone Kal-1; Dako, Carpinteria, CA) was used to test for virus-infected human MDM. For each mouse, $30-100$ serial (5- $\mu$ m-thick) sections were cut from the injection site and 3-7 sections (10 sections apart) analyzed. Antibodies to vimentin intermediate filaments (clone VIM 3B4; Boehringer Mannheim, Indianapolis, IN) were used for detection of human cells in mouse brains. Mouse microglia were detected by Griffonia simplicifolia lectinisolectin $\mathrm{B}_{4}$ labeling (lectin, G. simplicifolia; Vector Laboratories, Burlingame, CA) and astrocytes were detected by antibodies for glial fibrillary acidic protein (GFAP; Dako). Neuron-specific nuclear protein (NeuN), microtubule-associated protein 2 (MAP-2) (both from Chemicon, Temecula, CA) and heavy chain $(200 \mathrm{kDa})$ neurofilaments (Dako) were used for detection of neurons. Appropriate biotinylated secondary anti- bodies and the Vectastain Elite ABC kit (Vector Laboratories) were used to complete the immunohistochemical tests. All sections were counterstained with Mayer's hematoxylin. The numbers of human MDM and HIV-1 p24 antigen-positive cells were counted with a Nikon MicrophotFXA microscope. All obtained images were imported into Image-Pro Plus, version 4.0 (Media Cybernetics, Silver Spring, MD) for quantifying levels of GFAP-, lectin-, MAP-2-, and NeuN-positive staining.

Double-immunofluorescence staining was performed using Alexa Fluor 488 (green) and 594 (red) as a secondary antibody (Molecular Probes, Eugene, OR). Confocal laser-scanning imaging was used (Chung et al., 2002) to assess colocalization of the MAP-2 (neural cell bodies, axons, and dendrites) and human MDM (clone Ham56; Dako). The confocal system used for these studies was a Zeiss (Goettinger, Germany) LSM 410 laser-scanning microscope.

Western blot tests. Two millimeter brain tissue sections that included the injection line were removed for protein extractions. Parallel areas on the contralateral (noninjected) hemisphere were extracted and served as additional internal controls, which were used in comparison with corresponding tissues recovered from the injection sites. This data generated ratios between injected and contralateral hemispheres. Brain sections were homogenized in lysis buffer (50 mm Tris- $\mathrm{HCl}, \mathrm{pH} 7.4,150 \mathrm{~mm}$ $\mathrm{NaCl}, 5 \mathrm{~mm}$ EDTA, 1\% NP40, aprotinin, bestatin, leupeptin, pepstain A, aminoethyl benzenesulfonylfluoride, and E-64), and total protein concentration was determined by biocinchonic acid (BCA) assay. Equal amounts of protein were electrophoretically separated on SDSpolyacrylamide gels, transferred onto polyvinylidene difluoride membranes. Each membrane was incubated with a primary antibody to MAP-2 (Chemicon), tau 5 (BD Biosciences, San Jose, CA), phospho-tau Ser $^{202}$, phospho-tau $\mathrm{Thr}^{181}$, phospho- $\beta$-catenin, $\beta$-catenin (all from Sigma), phosphorylated neurofilament (Dako), or $\beta$-actin (Chemicon). Appropriate horseradish peroxidase-conjugated secondary antibodies were used. Membranes were treated with a chemiluminescent substrate to develop the signal and exposed to $\mathrm{x}$-ray film. Images were digitized using a Molecular Dynamics (Sunnyvale, CA) densitometer.

HIV RNA levels detected by real time PCR. The levels of HIV RNA in the injected hemisphere were determined by real time PCR with ABI 7000 prism (Perkin-Elmer, Applied Biosystems, Foster City, CA). Briefly, RNA was extracted from the brain tissues. The primers and probe used were as previously described (Cota et al., 2000) and are: forward, 5' - ACA TCA AGC CAT GCA AAT -3'; reverse, 5' - ATC TGG CCT GGT GCA ATA GG - $3^{\prime}$; and probe (FAM), 5' - CAT CAA TGA GGA AGC TGC AGA ATG GGA TAG A -3' (TAMRA). The reverse primer was used to make cDNA from RNA that was further amplified using primers and probe at $50^{\circ} \mathrm{C}$ for $2 \mathrm{~min}, 95^{\circ} \mathrm{C}$ for $10 \mathrm{~min}$, and 40 cycles at $95^{\circ} \mathrm{C}$ for $15 \mathrm{sec}$ and $60^{\circ} \mathrm{C}$ for 1 min. DNA from $8 E 5$ cells, which contain one integrated copy of proviral DNA per cell, was used to prepare the standard curve. Separate GAPDH amplifications were used as an endogenous control to ensure that equal amounts of RNA were used. Results are expressed as mean copy number \pm SEM. Data was analyzed using Excel (1994; Macintosh) with Student's $t$ test for comparisons.

\section{Results}

\section{Immunopathology}

Human HIV-1 $1_{\mathrm{ADA}}$-infected MDMs were stereotactically injected into the basal ganglia of SCID mice. Histopathological changes observed in murine brain tissue paralleled those seen for human HIVE as previously described (Persidsky et al., 1996). This included HIV-1 infection in perivascular and parenchymal human MDM, the formation of multinucleated giant cells, astrocytosis, and neuronal dropout. We identified and quantified the mean number of human MDM distributed through the injected hemisphere by immunostaining with vimentin in serial $5 \mu \mathrm{m}$ brain slices (total cells per section). The average numbers were determined using a total of 30 fields in 15 sections, from three sections per mouse and five mice per group (Fig. 1). At day 7 after injection, the mean number of MDMs were $83.8 \pm 6.5$ per section in HIVE mice with a control vehicle and 107.2 \pm 11.1 per section in 
HIVE-mice treated with VPA. At day 14 the number of MDM decreased in both control vehicle $(39.9 \pm 4.1$ per section $)$ and VPA-treated $(42.8 \pm 6.3$ per section) animals. By day 21, the number of MDM further decreased in both control vehicle $(5.0 \pm 4.2$ per section) and VPA-treated $(6.7 \pm 3.7$ per section $)$ mice. Real time PCR tests used to quantitate levels of HIV-1 RNA showed no differences between VPA-treated and control mice through all time points (data not shown).

We next quantified the absolute number of MDMs infected with HIV-1 by p24 antigen immunostaining. On day 7, 80\% of human MDMs in brains of VPA-treated and control mice were HIV-1 p24 antigenpositive. This increased to $\sim 95 \%$ at days 14 and 21. In control vehicle-treated mice compared with VPA-treated, the number of HIV-1 p24 antigen-positive MDMs per section were $62.2 \pm 8.5$ and $78.8 \pm 5.7$ on day $7,35.2 \pm 7.7$ and $40.0 \pm 2.2$ on day 14 , and $4.3 \pm 1.5$ and $6.2 \pm 2.5$ on day 21 . These results showed that the absolute number of HIV-1 p24-positive cells decreased in parallel to the loss of human MDMs.

Morphological changes in astrocytes were identified by immunostaining for GFAP antigen expression. We have shown the early manifestation of GFAP expression soon after implantation of HIV-1-infected MDMs into SCID mouse brains. Typically, GFAP-positive astrocytes are hypertrophied with a reactive morphology in the injected hemisphere. Cells with these properties were observed in both VPA-treated and control vehicle-treated mice when compared with sham injected (media without MDM) (Fig. 2). We quantified GFAP-positive astrocytes using ImagePro Plus, version 4.0 on serial coronal brain sections. Thirty fields (two fields per section, magnification $\times 200$ ) were scanned for 15 sections in five mice per group; these sections were collected from the injection line through basal ganglia and cerebral cortex. GFAP expression was quantified by determining the GFAPpositive area as a percentage of the total image area per microscopy field and calculated for a $0.5 \mathrm{~mm}$ window of tissue immediately surrounding the injection site. The percentage of GFAP-positive area (index) was $6.3 \pm 0.6$ compared with $1.2 \pm 0.4$ on day $7,12.7 \pm 0.8$ compared with $0.5 \pm 0.2$ on day 14 , and $1.1 \pm 0.5$ on day 21 in HIVE versus sham-operated mice. For control vehicle-treated and VPA-treated HIVE mice, the GFAP index was $6.3 \pm 0.6$ and $6.0 \pm 0.5$ at day 7 , $12.7 \pm 0.8$ and $12.4 \pm 1.0$ at day 14 , and $8.3 \pm 1.5$ and $8.5 \pm 1.2$ at day 21 . We next stained serial brain sections with $G$. simplicifolia lectin-isolectin $\mathrm{B}_{4}$ to determine activation of microglia. Microglial activation (as reflected by the presence of large ramified lectin-immunopositive cells) was detected in and around human MDMs in HIVE mice (Fig. 2). No such activation was detected in sham-operated animals. Using Image-Pro Plus, version 4.0, morphometric analysis determined the lectin-positive microglial area (calculated as a percentage of the area of the entire microscopy field) was $4.8 \pm 0.4$ and $4.4 \pm 0.2$ at day $7,9.1 \pm 0.4$ and $8.4 \pm 0.4$ at day 14 , and $11.9 \pm 0.1$ and $12.1 \pm 0.6$ at day 21 for control vehicle-treated and VPAtreated HIVE mice. The staining intensity for lectin-positive cells in affected brain areas significantly increased in HIVE compared with sham-operated mice $(p<0.01)$. There were no significant differences between VPA-treated and control HIVE animals.

VPA promotes neurite outgrowth and protects cultured neurons against the pro-apoptotic effects of a range of HIV-1 neurotoxins, including HIV-1 gp120. As the first step, we investigated the effects of VPA on neuronal survival after HIV-1 envelope protein glycoprotein (HIV-1 gp120) exposure to rat cortical neurons (RCNs). In these experiments RCNs were cultivated in neurobasal media with or without $3 \mathrm{mM}$ VPA in the presence or absence of $5 \mathrm{~nm}$ HIV-1 gp 120. Cultures were maintained for up to $5 \mathrm{~d}$. The cells were fixed in situ, and immunostaining was performed with antibodies to MAP-2 (Fig. 3). In RCNs propagated in neurobasal media, cells were distributed throughout the culture dish and connected one with the other. A high density of dendritic nodes and long neuritic processes were seen together with prominent cell bodies. This morphology remained constant with or without VPA (Fig. 3A,B). After HIV-1 gp120 exposure, neurons (Fig. 3C) displayed a low density of dendritic nodes, shorter neurites, and a loss of connected processes. Notably, VPA reversed these neurotoxic effects. In VPA-treated and HIV-1 gp120 exposed RCN neurites and dendritic nodes were visualized with long processes in high density at similar levels as present in controls. These observations were present in up to $5 \mathrm{~d}$ of VPA treatment and HIV-1 gp120 exposure to the RCN (Fig. 3D). Here many of the neurites connected with one another and prominent cell bodies were seen. Taken together, these findings demonstrated a major effect of VPA for protecting RCN against HIV-1 gp120 toxicity.

To best visualize changes in neuritic processes in HIVE mice and the effects by VPA treatment we used confocal microscopy. In these experiments double immunostaining with antibodies to MAP-2 and vimentin were used to determine the relationships between neurites and human MDMs. In HIVE animals neuronal loss extended beyond the areas of MDMs (Fig. $4 B$ ), and signifi- 


\section{control}

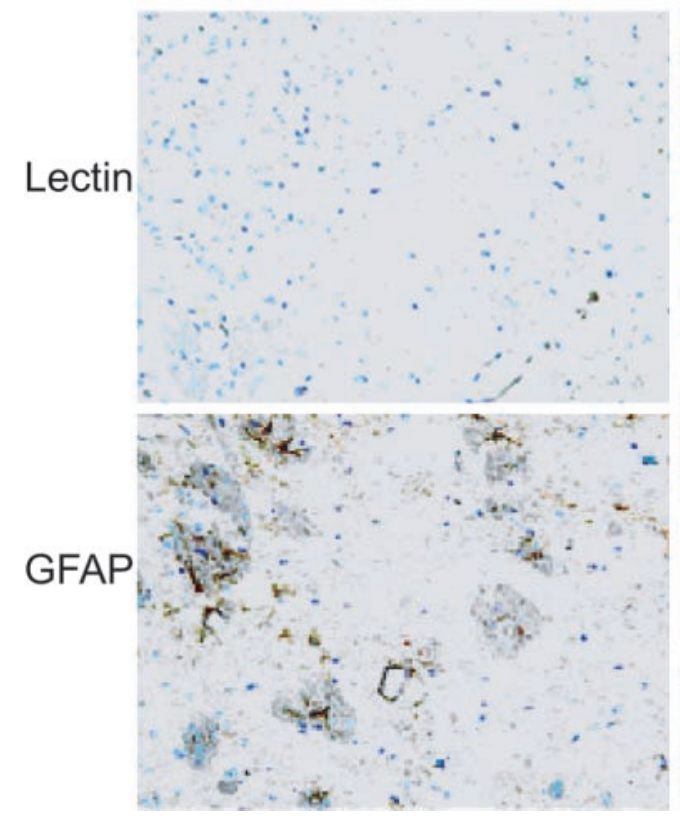

HIV-1

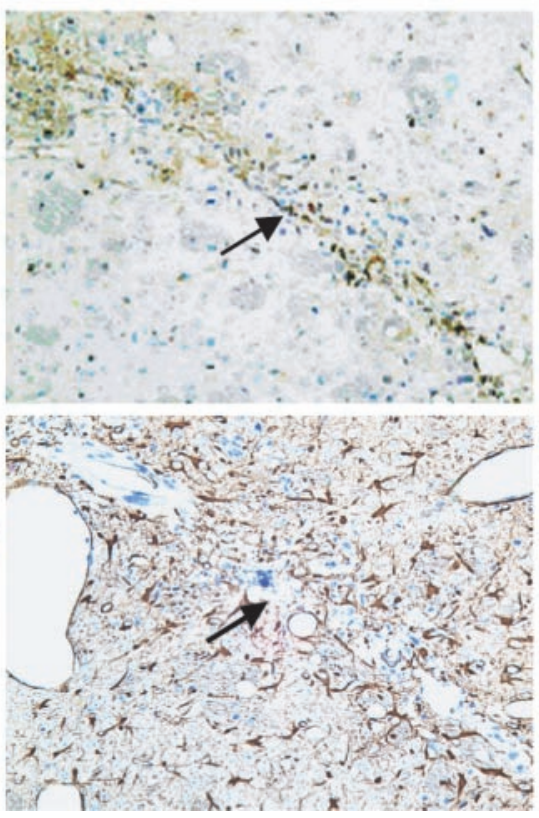

HIV-1+VPA

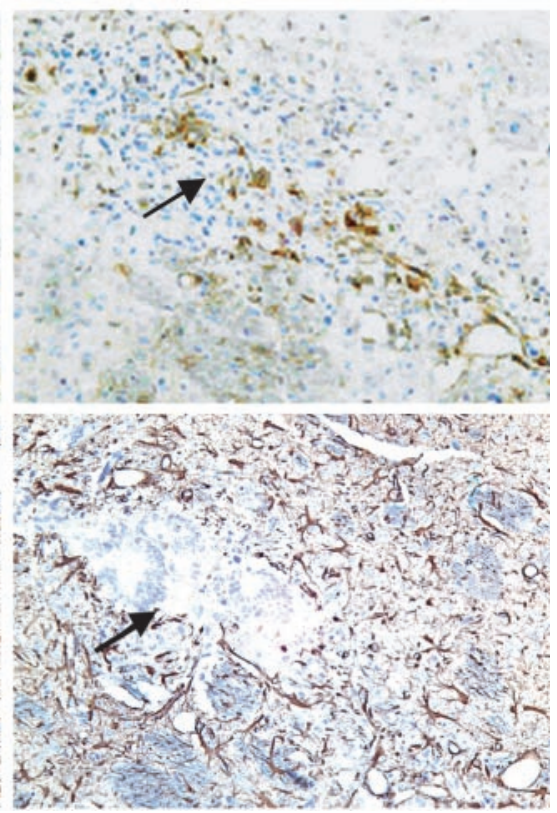

Figure 2. Astrocytes and microglial reactions in HIVE mice. Serial $5 \mu \mathrm{m}$ paraffin-embedded coronal brain sections were prepared from the MDM injection site. GFAP immunoreactivity reflects astrocyte responses to HIV-1-infected MDMs in HIVE mice after VPA treatment. Widespread astrocyte reactions surround the site of HIV-1-infected human MDMs. The area occupied by activated astrocytes was computed in a $0.5 \mathrm{~mm}$ window from the injection site. G. Simplicifolia lectin-isolectin $\mathrm{B}_{4}$ (Lectin) reflects microglial activation. The lectin-positive cells were seen only in the area of HIV-1-infected MDMs. No significant differences were observed in the area of GFAP-and lectin-positive cells between control HIVE and VPA-treated animals at all time points. Original magnification, $\times 200$.

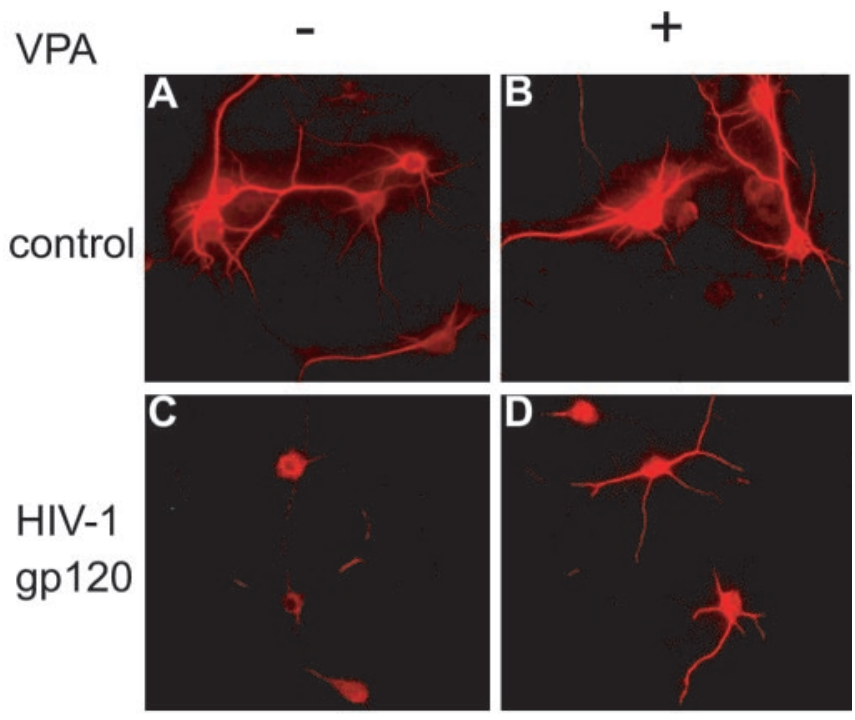

Figure 3. VPA and HIV-1 gp120 neurotoxicity. RCN were cultured in neural basal media $(A)$, with VPA (B), with HIV-1 gp120 ( $)$, and with HIV-1 gp120 and VPA (D). MAP-2-labeled neurons and neuronal processes are shown by immunofluorescent labeling. In neural basal media or in the presence of VPA alone, a high density of dendritic nodes and long neurite processes were observed with prominent cell bodies $(A, B)$. After HIV-1 gp120 exposure $(C$ neurons showed a lower density of dendritic nodes, shorter neurites, and process loss. VPA treatment reversed the neurotoxic process of HIV-1 gp120 exposure and showed long processes at high density $(D)$. Original magnification, $\times 400$.

cant neurite loss was present throughout the basal ganglia and cerebral cortex when compared with sham-operated mice (Fig. $4 A$ ). Most human MDMs were labeled by both MAP-2 and vimentin, indicating phagocytosis of degenerating neurites. Importantly and substantiating our previous experiments, the VPA- treated HIVE mice showed significantly higher levels of MAP-2positive neurites around the sites of HIV-1-infected MDMs (Fig. $4 C)$. In VPA-treated mice few human MDMs were doublelabeled by MAP-2 and vimentin, and more vimentin-positive cells were retained around the injection site. Visual examination of immunofluorescent stained sections showed clearly that VPA positively affected neuronal survival and process formation in HIVE mice. To assess whether VPA could protect neurons in HIVE mice, we first quantitated the levels of neurodegeneration in untreated animals. Neuropathological analyses were performed 7, 14, and $21 \mathrm{~d}$ after the injection of human MDM. Neuronal damage was easily seen and included shrunken and pyknotic nuclei and cell loss. To quantify the degree of cell loss, the neuronal nucleus, dendrites, and processes were assessed by immunostaining using antibodies to NeuN and MAP-2 (Fig. 5). The loss of positive neuronal staining corresponded to the area occupied by activated astrocytes and microglia cells and HIV-1infected human MDMs at day 7. Minimal restitution of neuronal loss was seen at day 14 . At day 21 the activated astrocytes and microglia and HIV-1-infected human MDMs were prominent. Minimal neuronal degeneration was observed in sham-operated animals. The data were obtained by quantified image analysis (Image-Pro Plus, version 4.0). The percentage area of MAP-2and NeuN-positive staining in injured tissue was $25.7 \pm 5.0$ area per field versus $6.1 \pm 1.3$ area per field at day 7,26.3 \pm 4.0 area per field versus $1.6 \pm 0.4$ area per field at day 14 , and $25.0 \pm 6.2$ area per field versus $3.7 \pm 1.3$ area per field at day 21 in sham as compared with HIVE mice. The differences in MAP-2- and NeuN-positive area between the two groups were statistically significant $(p>0.001)$. Compared with control vehicle-treated mice, importantly, VPA-treated HIVE mice showed significantly increased levels of MAP-2 and NeuN, and the percentage area of staining was $13.9 \pm 2.8(p<0.02), 7.6 \pm 2.8(p<0.04)$, and 
control

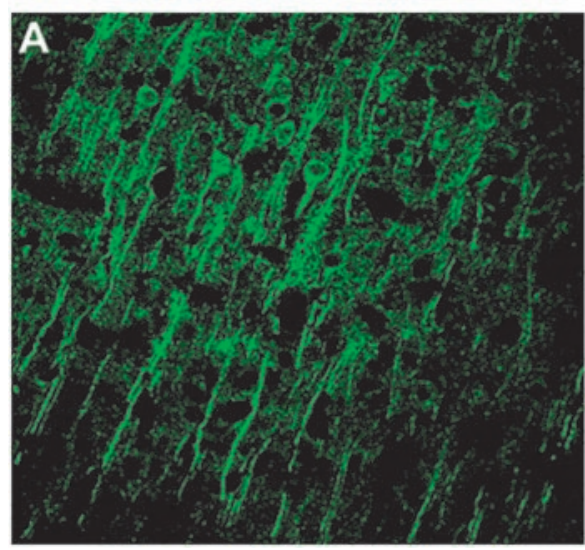

HIV-1

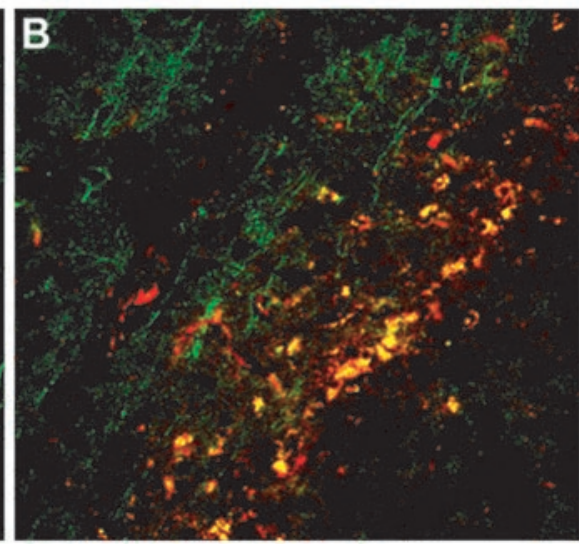

HIV-1+VPA

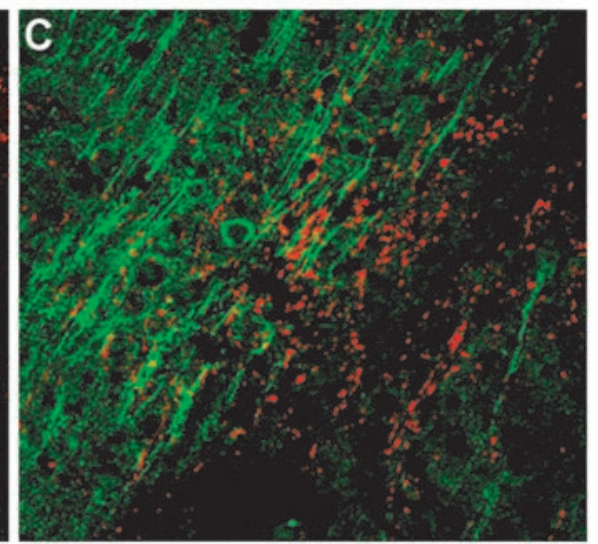

Figure 4. VPA-induced neuroprotection in HIVE mice. Immunofluorescence staining was performed in brains of HIVE mice after VPA treatment. Thirty-micrometer-thick sections of frozen brain tissue at the HIV-1-infected MDM injection site were cut and stained with antibodies to MAP-2 (green) and human vimentin (red). This allowed visualization of both neurites and human MDM. Tissues were visualized by laser confocal microscopy in the area of injection. Brain tissue from control sham-operated animals $(A)$, from the site of MDM injection in HIVE mice ( $B$ ), and from HIVE mice treated with VPA ( $C$ ) are shown. Original magnification, $\times 400$.

$15.6 \pm 2.1(p<0.01)$ on days 7,14 , and 21 . The results also showed a significant decrease in the level of MAP-2-positive dendrites and neurites compared with sham mice at days $7(p<0.04)$ and 14 $(p<0.01)$ but higher level of MAP-2positive dendrites and neurites occurred at day $21(p>0.1)$ (Fig. 5).

Neurofilament (NF) proteins belonging to the family of intermediate filaments were next examined. The heavy chain (200 $\mathrm{kDa}) \mathrm{NF}$ antibody recognizes neurofilament and phosphorylated neurofilament (NF-H) proteins. This antibody stained axons and neuronal cell bodies undergoing degeneration. NF-H-positive staining was seen in neuronal cell bodies in the basal ganglia around the HIV-1-infected MDMs (Fig. 6B,C, arrow). Importantly, NF-H staining was not observed in neuronal cell bodies of sham-operated animals (Fig. $6 A, D)$. In HIVE mice, NF-H-positive cell

bodies were observed with the highest intensity of staining around the injection site (Fig. 6B,E). Few NF-H-immunostained neuronal bodies were seen in VPA-treated HIVE animals (Fig. 6C,F).

\section{Mechanisms of VPA neuronal protection}

The neuronal protective efficiency of VPA was confirmed by Western blotting. The distribution of MAP-2- and NeuN-

immunoreactive neurons was analyzed by quantified immunoblots in brain tissues. The level of MAP-2 showed a significant decrease (Fig. 7A,B) in HIVE mice at days 7 and $14(p<0.01)$, and $21(p<0.02)$ compared with sham-operated mice. In contrast to control untreated HIVE mice, a significantly higher level of MAP-2 (Fig. 7B) was observed in VPA-treated mice at days 14 and 21 (Fig. $7 A)(p<0.05)$. There was no significant difference seen at day $7(p<0.03)$. VPA neuroprotection was observed through all time points. Results showed no significant MAP-2positive dendrites or neurite loss in VPA-treated mice at day 21 compared with sham-operated animals.

The levels of phosphorylated $\beta$-catenin were analyzed by quantified Western blotting to determine the effect on neuronal degeneration. $\beta$-catenin is a component of cell-to-cell adherent junctions and promotes transcription of target genes. The level of $\beta$-catenin is the biological marker of GSK-3 activation. We determined the level of $\beta$-catenin by Western blot in mice brain tissue (Fig. $8 C$ ). Whereas the distribution of $\beta$-catenin was stable over time in sham mice, significantly higher levels of $\beta$-catenin were present in VPA-treated HIVE mice compared with untreated HIVE mice only at day 14 (Fig. $8 A)(p<0.05)$. We next determined the level of phosphorylation of $\beta$-catenin by Western blot using anti-phospho- $\beta$-catenin-Ser ${ }^{33,37}$. Quantitative immunoblot analysis (Fig. $8 \mathrm{~B}$ ) showed HIVE mice had significantly increased levels of phosphorylated $\beta$-catenin $(p<0.05$ at day 7 , $p<0.01$ at days 14 and 21) compared with sham mice. In VPAtreated HIVE mice, phosphorylated $\beta$-catenin was increased at days 7 and $14(p<0.05)$, but no significant difference was seen at day $21(p>0.1)$ compared with sham. VPA, therefore, appeared to block $\beta$-catenin phosphorylation in HIVE mice after longterm treatment. Although the reduction of $\beta$-catenin phosphorylation was not observed in VPA-treated mice at day 7 , significant 


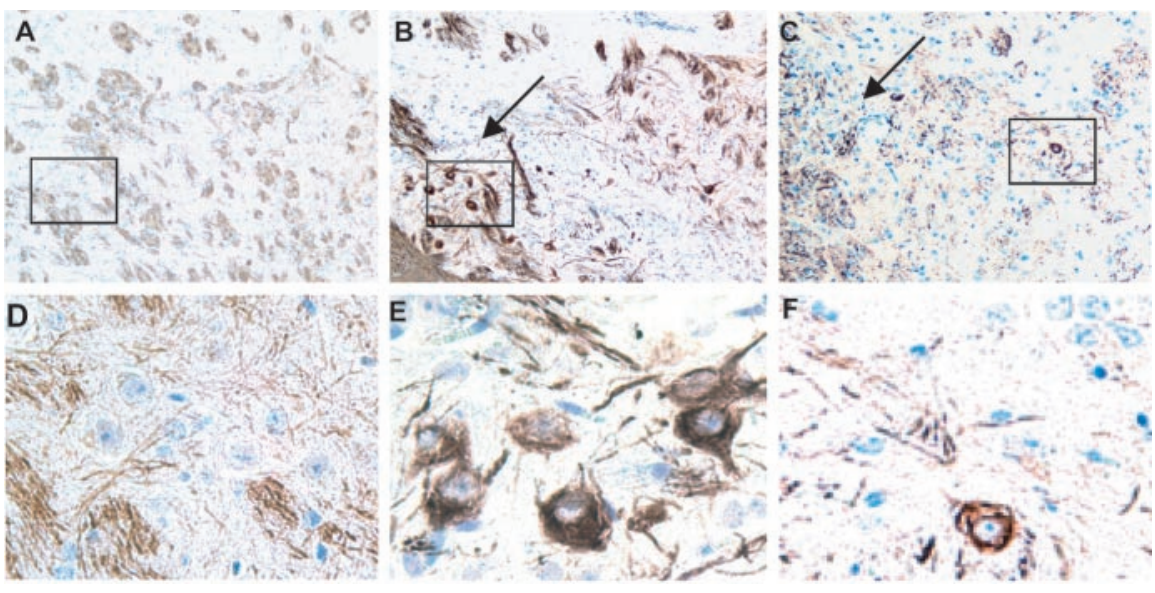

Figure 6. VPA alters neuronal expression of NF-H in HIVE mice. Serial $5 \mu \mathrm{m}$ sections were prepared from paraformaldehydefixed paraffin-embedded tissue. Brain tissue was examined in the area of the basal ganglia, the region of HIV-1 infected MDM ( $B_{\text {, }}$ $C$, arrow). Sections were stained with antibodies to heavy chain $(200 \mathrm{kDa}$ ) neurofilament (including nonphosphorylated and phosphorylated neurofilament). NF-H staining was not observed in neuronal cell bodies in sham-operated mice $(A, D)$. However, $\mathrm{NF}-\mathrm{H}$-positive neuronal cell bodies were commonly observed in the area of HIV-1-infected human MDMs $(B, E)$. NF-H expression within the neuronal body was decreased in VPA-treated HIVE mice $(C, F)$. The high-powered views shown in $D-F$ are enlargements of the insets shown in $A-C$. All tissue sections were counterstained with Mayer's hematoxylin. Original magnification: $A-C$, $\times 200 ; D-F, \times 600$.

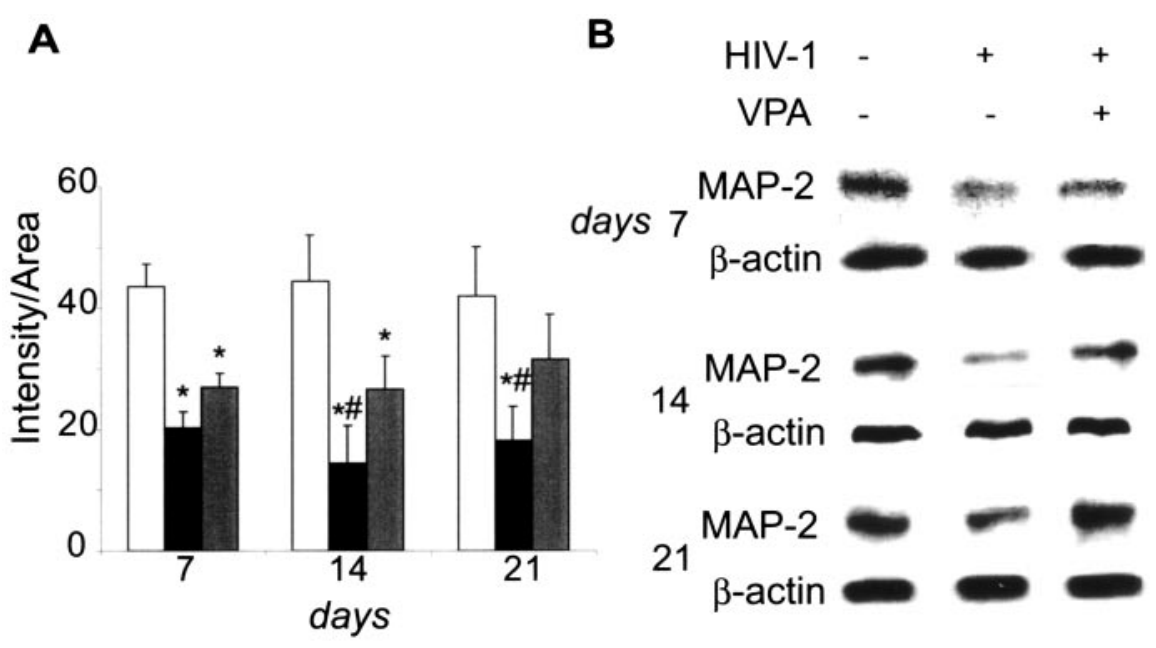

Figure 7. Levels of neuronal antigens in murine HIVE. Neuronal degeneration and VPA protection was analyzed by Western blots using antibody for MAP-2 (B). A, Decreased MAP-2 antigens are shown in brains of HIVE (black bar) as compared with sham-operated mice (white bar). VPA-treated animals showed (gray bar) increased MAP-2 at day 7. Significant differences were only seen on days 14 and 21. Quantitation of the blots showed MAP-2 antigens significantly lower in HIVE animals as compared with sham-operated mice at days 7-14 $(p<0.01)$ and 21 ( $p<0.02$ ). VPA-treated mice showed higher levels of MAP-2 compared with HIVE mice at day $7(p<0.03)$ and significantly on days 14 and $21(p<0.05)$ (* vs control and \# vs VPA).

reduction in $\beta$-catenin phosphorylation was present at days 14 $(p<0.05)$ and $21(p<0.01)$ compared with HIVE mice.

The overexpression of phosphorylated tau is a neuropathological hallmark of neurodegeneration. Quantitation of the levels of total tau (Fig. $9 A$, blot shown in $D$ ) by anti-tau 5 showed no difference between sham and HIVE mice $(p>0.05)$. We next performed immunoblot analysis with antibodies specific for tau phosphorylation at $\mathrm{Ser}^{202}$ and $\mathrm{Thr}^{181}$ (Fig. 9D). In contrast with total tau, the levels of phosphorylated tau at both sites increased in HIVE animals. Quantification of the blots showed increased level of phospho-tau Ser ${ }^{202}$ after HIV-1 infected human MDM injection (Fig. $9 B)$ at all time points $(p<0.01)$. VPA-treated HIVE mice also showed a higher distribution of phospho-tau $\mathrm{Ser}^{202}$ compared with sham mice $(p<0.05)$. However, VPA significantly decreased the phosphorylation of tau at Ser ${ }^{202}$ compared with untreated HIVE animals at all time points $(p<0.05$ at days 7 and $14, p<0.01$ at day 21). We also tested phosphorylated tau Ser ${ }^{404}$ with all cases, but no significant differences were observed in sham, HIVE, and VPA-treated mice (data not shown). Analysis of the level of phosphorylated tau at $\mathrm{Thr}^{181}$ (Fig. 9C) showed significant increase in phospho-tau Thr ${ }^{181}$ at days 7 and $14(p<0.05)$, which returned to background level by day $21(p<0.3)$ in HIVE mice. This pattern was seen neither in VPA-treated HIVE animals nor sham mice. VPA showed a trend of decreased levels of hyperphosphorylated tau, however, only day 14 was significant $(p<$ 0.05 ) as compared with HIVE mice. This result indicated that the different phospho-tau sites had a different response in HIVE SCID mice. VPA continuously decreased the overphosphorylation of tau $\mathrm{Ser}^{202}$, whereas phosphorylated tau $\mathrm{Thr}^{181}$ returned to background levels by day 21.

\section{Discussion}

Using both in vitro and in vivo model systems reflective of human HIVE, we demonstrated that VPA protects neurons against the secretory neurotoxins produced by virus-infected macrophages. VPA promoted neuronal survival and inhibited neurotoxicity induced by viral and cellular macrophage factors. In our in vitro model of HIVE, RCN exposed to HIV-1 gp120 displayed a low density of dendritic nodes, shorter neurites, and a loss of neuronal connections. These effects were blocked by VPA. Indeed, VPA-treated gp120-exposed RCN contained readily detectable MAP-2-positive neurites and an increased density of dendritic nodes. Moreover, in our in vivo studies, VPA treatment of HIVE mice resulted in the preservation of MAP-2-positive dendrites and axons and an increase in $\mathrm{NeuN}$ antigen-positive nuclei, as compared with untreated animals. The neuroprotective actions of VPA were also demonstrated by its abilities to inhibit neurofilament expression in neuronal cell soma. These findings, taken together, suggest that VPA may ameliorate HIVE.

It has long been appreciated that HIV-1 infection of the CNS is associated with neocortical and dendritic damage (Wiley et al., 1991b; Masliah et al., 1992b). Disease pathogenesis revolves around the continuous ingress of monocytes into the brain with subsequent immune activation and an expanding viral reservoir in brain microglia and perivascular macrophages (Wiley et al., 1991a; Hori et al., 1999; Weiss et al., 1999). A murine model of HIVE was generated to reflect the neurotoxicity and neuropathogenesis of HIV-1 infection (Persidsky et al., 1996; Sanders et al., 1998; Limoges et al., 2000). We confirmed that the histological 
A

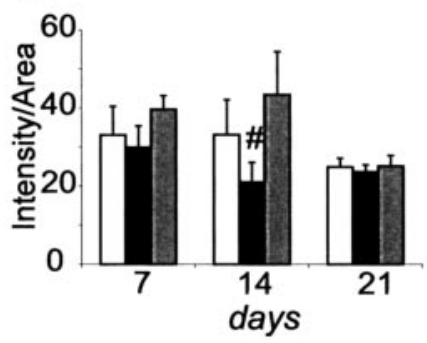

C

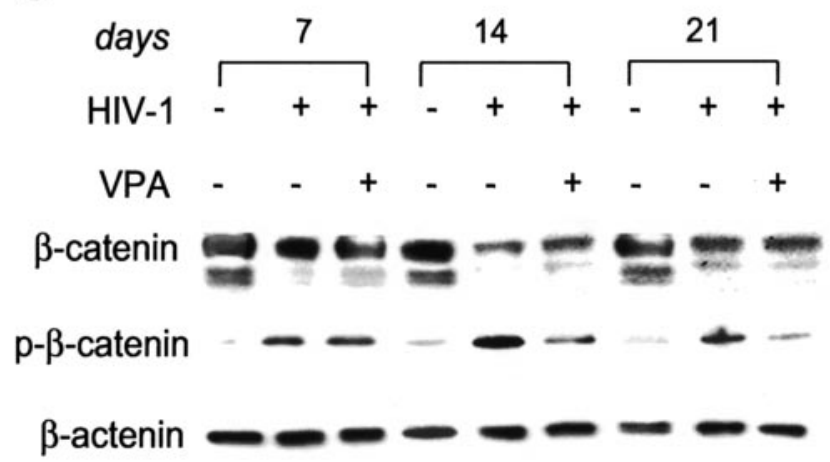

Figure 8. VPA regulates $\beta$-catenin expression in HIVE mice. Levels of $\beta$-catenin and phosphorylated $\beta$-catenin were assessed by Western blot ( $($ ). Quantitation of the blots showed a decrease level of $\beta$-catenin in HIVE mice (black bar) as compared with sham (white bar). VPAtreated HIVE mice (gray bar) had higher levels of $\beta$-catenin when compared with untreated HIVE animals $(A)$, with a significant increase observed at day $14(p<0.05)$. Phosphorylated $\beta$-catenin antigens were increased in HIVE animals (black bar) $(p<0.05$ at day $7, p<0.01$ at days 14 and 21) compared with sham (white bar). VPA treatment of HIVE mice (gray bar) showed a reduction in the phosphorylated $\beta$-catenin at days 14 and $21(p<0.05)$ as compared with untreated HIVE animals. The level of phosphorylated $\beta$-catenin in VPA-treated animals was no different from sham at day 21 ( $p>0.1$ ) ( ${ }^{*}$ vs control and \# vs VPA).

presence of encephalitis is a major viral-associated inflammatory response to HIV-1-infected MDMs in brain. This response, however, is not relevant to the amount of virus and number of MDMs because results showed a large decrease of HIV-1-infected macrophages on days 14 and 21 after injection. This suggests that neuronal damage in HIV-1 infection may be secondary to the shedding of viral-associated factors or release of neurotoxic products from infected macrophages.

Improvement in cognitive function and restitution of neuronal injury involve control of virus, inhibition of inflammatory activities, and neuronal protection (Schielke, 1993; Persidsky and Gendelman, 2002). Neurodegeneration is controlled in significant measure by PCAT, but not eliminated by it, and virus often persists in the brain as a continuous reservoir representing a nidus of infectious virus. However, this is not operative for VPA. How VPA affects neuronal protection in HIVE without altering the levels of macrophage HIV-1 infection or inhibiting the neurotoxic activities remains unknown. In this regard VPA provides a model of novel pathways for neuronal protection against the viral and cellular neurotoxins secreted by HIV-1-infected macrophages. Data from both our in vitro and in vivo model systems of HIVE strongly suggest that VPA-mediated neuroprotection involves the inhibition of GSK-3 $\beta$ (Maggirwar et al., 1999; Tong et al., 2001). This is consistent, for example, with the inhibition of phosphorylation of both $\beta$-catenin $\left(\operatorname{Ser}^{33,37}\right)$ and tau $\left(\operatorname{Ser}^{202}\right.$ and $\mathrm{Thr}^{181}$ ), as well as with the overall increase in total $\beta$-catenin protein levels. Hyperphosphorylation of $\beta$-catenin and Tau can in fact directly affect neuronal apoptosis and dysfunction (Ferrer
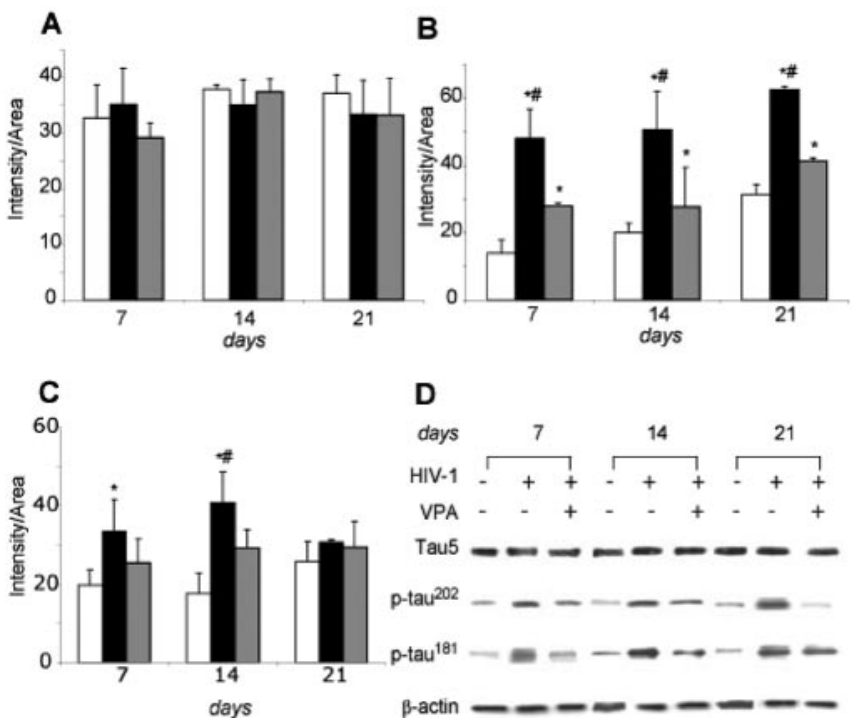

Figure 9. VPA regulates levels of phosphorylated tau. Western blotting was performed with antibodies to total tau (anti-tau 5) and phosphorylated tau (anti-tau pSer ${ }^{202}$ and pThr ${ }^{181}$ ) (D). Quantitation showed no significant difference in the level of total tau antigens in mouse brains $(A)$ between all groups (sham with white bar, HIVE with black bar and VPA with gray bar). A higher amount of phosphorylated tau-Ser ${ }^{202}$ was present in the HIVE animals (black bar) $(p<$ 0.01 ) as compared with sham (white bar) for all time points ( $B)$. VPA treatment (gray bar) significantly reduced the level of phosphorylated tau-Ser ${ }^{202}(p<0.05)$ compared with the untreated HIVE animals ( $B$ ). Phosphorylated tau Thr ${ }^{181}$ was increased at days 7 and $14(C)$ in HIVE animals (black bar) as compared with sham (white bar). VPA (gray bar) treatment significantly reduced the level of phospho-tau Thr ${ }^{181}$ at day $14(p<0.05)$ as compared with untreated mice ( $C$ ( ${ }^{*}$ vs control and \# vs VPA).

et al., 2003; Hong et al., 2003; Kobayashi et al., 2003). $\beta$-catenin levels are markedly reduced in some brain degenerative diseases, and loss of $\beta$-catenin signaling seems to increase neuronal vulnerability to apoptosis. Originally identified as a regulator of glycogen synthesis, GSK- $3 \beta$ also plays an important role in affecting proapoptotic factors that contribute to neuronal loss (Bhat et al., 2000; Hashimoto et al., 2002; Stoica et al., 2003).

Consistent with this view, $\beta$-catenin has been shown to affect neuronal development by influencing cell division, decreasing cell death, and inducing neurite outgrowth (Chenn and Walsh, 2002 ; Zechner et al., 2003). Thus, inhibition of GSK-3 $\beta$ may serve to offset the $\beta$-catenin destabilizing effects and thereby reduce the vulnerability of affected neurons to apoptosis. In our case, we find the hyperphosphorylation of $\beta$-catenin occurs in HIVE SCID mice brains concurrently with neuroglia immunoreactions and neuronal degeneration. Similarly, specific phosphorylated isoforms of Tau have been associated with neurodegenerative disorders (Feany et al., 1995), including Alzheimer's disease (Andreasen et al., 2003). Tau phosphorylation, a possible disorganization mechanism of the microtubule cytoskeleton, has been produced in GSK-3 $\beta$ conditional transgenic mice (Gotz and Nitsch, 2001). In our observation, highly phosphorylated Tau $\mathrm{Ser}^{202}$ and $\mathrm{Thr}^{181}$ occur during the neuronal injury in HIVE mice. Both Tau and $\beta$-catenin may represent important physiologic targets of GSK $-3 \beta$ that may contribute to neuronal loss and neuronal damage in the context of HAD. The results support the hypothesis that degradation of $\beta$-catenin and Tau phosphorylation might be a major event in the pathogenesis of HIVE or HAD and raise the possibility that VPA could inhibit the hyperphosphorylation of $\beta$-catenin and Tau through the regulation of GSK- $3 \beta$ to promote neuronal survival.

Astrocyte and microglial responses to HIV-1-infected macro- 
phages vary (Trillo-Pazos et al., 2003). Whereas microglia activation is seen only in close proximity to the injection site, astrocyte responses are more diffuse. In general, the degree of neuronal injury in our HIVE SCID model correlated with the degree of activation of microglia and astrocytes. However, neuronal loss occurred during the entire $21 \mathrm{~d}$ experimental period, even at late time points when most HIV-1-infected MDMs had been eliminated. This suggested that activation of glia in response to viral infection might serve to perpetuate neuronal injury. Data also demonstrated significant regional susceptibilities (H. Dou, unpublished observations) of neurons to the toxic effects of infected macrophages. Although focal regions within the basal ganglia were sensitive to viral and cellular neurotoxins, cortical neurons appeared more resistant. Furthermore, these observations are consistent with the effects that HIV has on NF expression in focal neuronal populations. We demonstrated that HIV-1 infection of MDM increases NF-H expression in the cell bodies of neurons contained in the basal ganglia and cerebral cortex. However, no NF-H-positive neurons could be demonstrated in the caudate and putamen. Thus, the interplay between the levels of viral infection, microglial, and astrocyte activation and regional neuronal susceptibility to injury may all play important roles in disease pathogenesis. These observations may help explain the specific neurological sequelae that often follow HIV-1 infection of the nervous system (Masliah et al., 1992a,b).

Increases in MAP-2 antigen staining, as a consequence of VPA treatment of HIVE mice is arguably an important signature for neuroprotection for a number of reasons. MAP-2 strongly parallels neuronal survival during development as well as neurodegeneration (San Jose et al., 1997; Marx et al., 2001; Coronas et al., 2002). MAP-2 also plays a pivotal role in polymerization of tubulin into microtubules and helps provide physical stability to microtubule formations. Loss of MAP-2 defines dendritic pathology (Saatman et al., 1998; Adamec et al., 2001). Thus, the protective effects of VPA on MAP-2 are consistent with its role in neuroprotection. One concern for translating these findings to clinical practice is whether VPA passage into the brain is facilitated or restricted. For example, pluronic P85, a well established inhibitor of $p$-glycoprotein, affects the transport of several antiretroviral drugs such as ritonavir. In contrast, $p$-glycoprotein does not affect transport of VPA across brain microvessel endothelial cells. This suggests that VPA is less affected by $p$-glycoprotein drug efflux transport systems than other studied drugs (Batrakova et al., 1999; Kabanov et al., 2002; Wang et al., 2003). The observations made in this report are significant because they show that neurons can be protected against HIV-1 independent of alterations in viral replication and inflammatory cell responses. Moreover, the results strongly support the importance of adjunctive therapies for treatment of HAD. The likelihood for clinical success is underscored by the fact that VPA exhibits limited side affects with long-term use and has shown clinical benefit (Turski et al., 1989; Chen et al., 2001).

\section{References}

Adamec E, Yang F, Cole GM, Nixon RA (2001) Multiple-label immunocytochemistry for the evaluation of nature of cell death in experimental models of neurodegeneration. Brain Res Brain Res Protoc 7:193-202.

Anderson E, Zink W, Xiong H, Gendelman HE (2002) HIV-1-associated dementia: a metabolic encephalopathy perpetrated by virus-infected and immune-competent mononuclear phagocytes. J Acquir Immune Defic Syndr [Suppl 2] 31:S43-S54.

Andreasen N, Vanmechelen E, Vanderstichele H, Davidsson P, Blennow K (2003) Cerebrospinal fluid levels of total-tau, phospho-tau and A beta 42 predicts development of Alzheimer's disease in patients with mild cognitive impairment. Acta Neurol Scand Suppl 179:47-51.

Batrakova EV, Li S, Miller DW, Kabanov AV (1999) Pluronic P85 increases permeability of a broad spectrum of drugs in polarized BBMEC and Caco-2 cell monolayers. Pharm Res 16:1366-1372.

Bhat RV, Shanley J, Correll MP, Fieles WE, Keith RA, Scott CW, Lee CM (2000) Regulation and localization of tyrosine216 phosphorylation of glycogen synthase kinase-3beta in cellular and animal models of neuronal degeneration. Proc Natl Acad Sci USA 97:11074-11079.

Chen G, Huang LD, Jiang YM, Manji HK (1999) The mood-stabilizing agent valproate inhibits the activity of glycogen synthase kinase-3. J Neurochem 72:1327-1330.

Chen G, Huang LD, Zeng WZ, H KM (2001) Mood stabilizers regulate cytoprotective and mRNA-binding proteins in the brain: long-term effects on cell survival and transcript stability. Int J Neuropsychopharmacol $4: 47-64$.

Chenn A, Walsh CA (2002) Regulation of cerebral cortical size by control of cell cycle exit in neural precursors. Science 297:365-369.

Chung MS, Pepose JS, El-Agha MS, Cavanagh HD, Al-Agha S (2002) Confocal microscopic findings in a case of delayed-onset bilateral diffuse lamellar keratitis after laser in situ keratomileusis. J Cataract Refract Surg 28:1467-1470.

Coronas V, Arnault P, Roger M (2002) Cortical diffusible factors increase MAP-2 immunoreactive neuronal population in thalamic cultures. Neurosci Res 43:57-67.

Cota M, Mengozzi M, Vicenzi E, Panina-Bordignon P, Sinigaglia F, Transidico P, Sozzani S, Mantovani A, Poli G (2000) Selective inhibition of HIV replication in primary macrophages but not $\mathrm{T}$ lymphocytes by macrophage-derived chemokine. Proc Natl Acad Sci USA 97:9162-9167.

Feany MB, Ksiezak-Reding H, Liu WK, Vincent I, Yen SH, Dickson DW (1995) Epitope expression and hyperphosphorylation of tau protein in corticobasal degeneration: differentiation from progressive supranuclear palsy. Acta Neuropathol (Berl) 90:37-43.

Ferrer I, Barrachina M, Tolnay M, Rey MJ, Vidal N, Carmona M, Blanco R, Puig B (2003) Phosphorylated protein kinases associated with neuronal and glial tau deposits in argyrophilic grain disease. Brain Pathol 13:62-78.

Gendelman HE (2002) Neural immunity: friend or foe? J Neurovirol $8: 474-479$.

Gendelman HE, Orenstein JM, Martin MA, Ferrua C, Mitra R, Phipps T, Wahl LA, Lane HC, Fauci AS, Burke DS, Skillman D, Meltzer MS (1988) Efficient isolation and propagation of human immunodeficiency virus on recombinant colony-stimulating factor 1-treated monocytes. J Exp Med 167:1428-1441.

Gendelman HE, Diesing S, Gelbard H, Swindells S (2003) The neuropathogenesis of HIV-1 infection. In: AIDS and other manifestations of HIV infection, Fourth Edition (Wormser GP, ed), Amsterdam: Elsevier, in press.

Genis PJM, Bernton EW, Boyle T, Gelbard HA, Dzenko K, Keane RW, Resnick L, Mizrachi Y, Volsky DJ, Epstein LG, Gendelman HE (1992) Cytokines and arachidonic metabolites produced during human immunodeficiency virus (HIV)-infected macrophage-astroglia interactions: implications for the neuropathogenesis of HIV disease. J Exp Med 176:1703-1718.

Gotz J, Nitsch RM (2001) Compartmentalized tau hyperphosphorylation and increased levels of kinases in transgenic mice. NeuroReport 12:2007-2016.

Hall AC, Brennan A, Goold RG, Cleverley K, Lucas FR, Gordon-Weeks PR, Salinas PC (2002) Valproate regulates GSK-3-mediated axonal remodeling and synapsin I clustering in developing neurons. Mol Cell Neurosci $20: 257-270$.

Hashimoto M, Sagara Y, Langford D, Everall IP, Mallory M, Everson A, Digicaylioglu M, Masliah E (2002) Fibroblast growth factor 1 regulates signaling via the glycogen synthase kinase-3beta pathway. Implications for neuroprotection. J Biol Chem 277:32985-32991.

Hong DJ, Pei AL, Sun FY, Zhu CQ (2003) Aberrant neuronal expression of mitotic protein, tau and Bax in the rat brain after injection of Abeta(2535 ) into the amygdala. Sheng Li Xue Bao 55:142-146.

Hori K, Burd PR, Furuke K, Kutza J, Weih KA, Clouse KA (1999) Human immunodeficiency virus-1-infected macrophages induce inducible nitric oxide synthase and nitric oxide (NO) production in astrocytes: astrocytic $\mathrm{NO}$ as a possible mediator of neural damage in acquired immunodeficiency syndrome. Blood 93:1843-1850. 
Illig AM, Melia K, Snyder PJ, Badura LL (2000) Sodium valproate alters GnRH-GABA interactions during development in seizure-prone mice. Brain Res 885:192-200.

Kabanov AV, Batrakova EV, Alakhov VY (2002) Pluronic block copolymers as novel polymer therapeutics for drug and gene delivery. J Control Release $82: 189-212$.

Kobayashi K, Nakano H, Hayashi M, Shimazaki M, Fukutani Y, Sasaki K, Sugimori K, Koshino Y (2003) Association of phosphorylation site of tau protein with neuronal apoptosis in Alzheimer's disease. J Neurol Sci 208:17-24.

Kravcik S, Gallicano K, Roth V, Cassol S, Hawley-Foss N, Badley A, Cameron DW (1999) Cerebrospinal fluid HIV RNA and drug levels with combination ritonavir and saquinavir. J Acquir Immune Defic Syndr 21:371-375.

Ledergerber BEM, Opravil M, Telenti A, Hirschel B, Battegay M, Vernazza P, Sudre P, Flepp M, Furrer H, Francioli P, Weber R (1999) Clinical progression and virlogical failure on highly active antiretroviral therapy in HIV-1 patients: a prospective cohort study. Swiss HIV Cohort Study. Lancet 353:863-868.

Limoges J, Persidsky Y, Poluektova L, Rasmussen J, Ratanasuwan W, Zelivyanskaya M, McClernon DR, Lanier ER, Gendelman HE (2000) Evaluation of antiretroviral drug efficacy for HIV-1 encephalitis in SCID mice. Neurology 54:379-389.

Lipton SA, Gendelman HE (1995) Seminars in medicine of the Beth Israel Hospital, Boston. Dementia associated with the acquired immunodeficiency syndrome. N Engl J Med 332:934-940.

Maggirwar SB, Tong N, Ramirez S, Gelbard HA, Dewhurst S (1999) HIV-1 Tat-mediated activation of glycogen synthase kinase-3beta contributes to Tat-mediated neurotoxicity. J Neurochem 73:578-586.

Manji HK, Moore GJ, Chen G (2000) Lithium up-regulates the cytoprotective protein $\mathrm{Bcl}-2$ in the CNS in vivo: a role for neurotrophic and neuroprotective effects in manic depressive illness. J Clin Psychiatry [Suppl 9] 61:82-96.

Marx CE, Jarskog LF, Lauder JM, Lieberman JA, Gilmore JH (2001) Cytokine effects on cortical neuron MAP-2 immunoreactivity: implications for schizophrenia. Biol Psychiatry 50:743-749.

Masliah E, Ge N, Achim CL, Hansen LA, Wiley CA (1992a) Selective neuronal vulnerability in HIV encephalitis. J Neuropathol Exp Neurol 51:585-593.

Masliah E, Achim CL, Ge N, DeTeresa R, Terry RD, Wiley CA (1992b) Spectrum of human immunodeficiency virus-associated neocortical damage. Ann Neurol 32:321-329.

O’Leary G, Bacon CL, Odumeru O, Fagan C, Fitzpatrick T, Gallagher HC, Moriarty DC, Regan CM (2000) Antiproliferative actions of inhalational anesthetics: comparisons to the valproate teratogen. Int J Dev Neurosci 18:39-45.

Perez M, Rojo AI, Wandosell F, Diaz-Nido J, Avila J (2003) Prion peptide induces neuronal cell death through a pathway involving glycogen synthase kinase 3. Biochem J 372:129-136.

Persidsky Y, Gendelman HE (2002) Murine models for human immunodeficiency virus type 1-associated dementia: the development of new treatment testing paradigms. J Neurovirol [Suppl 2] 8:49-52.

Persidsky YNH, Sasseville VG, Epstein LG, Gendelman HE (1995) The development of animal model systems for HIV-1 encephalitis and its associated dementia. J Neurovirol 1:229-243.

Persidsky Y, Limoges J, McComb R, Bock P, Baldwin T, Tyor W, Patil A, Nottet HS, Epstein L, Gelbard H, Flanagan E, Reinhard J, Pirruccello SJ, Gendelman HE (1996) Human immunodeficiency virus encephalitis in SCID mice. Am J Pathol 149:1027-1053.

Saatman KE, Graham DI, McIntosh TK (1998) The neuronal cytoskeleton is at risk after mild and moderate brain injury. J Neurotrauma 15:1047-1058.

Sacktor N, Lyles RH, Skolasky R, Kleeberger C, Selnes OA, Miller EN, Becker JT, Cohen B, McArthur JC (2001) HIV-associated neurologic disease incidence changes: multicenter AIDS Cohort Study, 1990-1998. Neurology 56:257-260.

San Jose I, Vazquez E, Garcia-Atares N, Huerta JJ, Vega JA, Represa J (1997) Differential expression of microtubule associated protein MAP-2 in de- veloping cochleovestibular neurons and its modulation by neurotrophin-3. Int J Dev Biol 41:509-519.

Sanders VJ, Mehta AP, White MG, Achim CL (1998) A murine model of HIV encephalitis: xenotransplantation of HIV-infected human neuroglia into SCID mouse brain. Neuropathol Appl Neurobiol 24:461-467.

Schielke E (1993) HIV encephalopathy: clinical aspects, neuropathology and pathogenesis. Nervenarzt 64:83-90.

Schifitto G, Kieburtz K, McDermott MP, McArthur J, Marder K, Sacktor N, Palumbo D, Selnes O, Stern Y, Epstein L, Albert S (2001) Clinical trials in HIV-associated cognitive impairment: cognitive and functional outcomes. Neurology 56:415-418.

Stern Y, McDermott MP, Albert S, Palumbo D, Selnes OA, McArthur J, Sacktor N, Schifitto G, Kieburtz K, Epstein L, Marder KS (2001) Factors associated with incident human immunodeficiency virus-dementia. Arch Neurol 58:473-479.

Stoica BA, Movsesyan VA, Lea IV PM, Faden AI (2003) Ceramide-induced neuronal apoptosis is associated with dephosphorylation of Akt, BAD, FKHR, GSK-3beta, and induction of the mitochondrial-dependent intrinsic caspase pathway. Mol Cell Neurosci 22:365-382.

Swindells S, Zheng J, Gendelman HE (1999) HIV-associated dementia: new insights into disease pathogenesis and therapeutic interventions. AIDS Patient Care STDS 13:153-163.

Tariot PN, Loy R, Ryan JM, Porsteinsson A, Ismail S (2002) Mood stabilizers in Alzheimer's disease: symptomatic and neuroprotective rationales. Adv Drug Deliv Rev 54:1567-1577.

Tong N, Sanchez JF, Maggirwar SB, Ramirez SH, Guo H, Dewhurst S, Gelbard HA (2001) Activation of glycogen synthase kinase 3 beta (GSK-3beta) by platelet activating factor mediates migration and cell death in cerebellar granule neurons. Eur J Neurosci 13:1913-1922.

Trillo-Pazos G, Diamanturos A, Rislove L, Menza T, Chao W, Belem P, Sadiq S, Morgello S, Sharer L, Volsky DJ (2003) Detection of HIV-1 DNA in microglia/macrophages, astrocytes and neurons isolated from brain tissue with HIV-1 encephalitis by laser capture microdissection. Brain Pathol 13:144-154.

Turski L, Ikonomidou C, Turski WA, Bortolotto ZA, Cavalheiro EA (1989) Review: cholinergic mechanisms and epileptogenesis. The seizures induced by pilocarpine: a novel experimental model of intractable epilepsy. Synapse 3:154-171.

Wang Y, Zhou D, Wang B, Li H, Chai H, Zhou Q, Zhang S, Stefan H (2003) A kindling model of pharmacoresistant temporal lobe epilepsy in Sprague-Dawley rats induced by Coriaria lactone and its possible mechanism. Epilepsia 44:475-488.

Weiss JM, Nath A, Major EO, Berman JW (1999) HIV-1 Tat induces monocyte chemoattractant protein-1-mediated monocyte transmigration across a model of the human blood-brain barrier and up-regulates CCR5 expression on human monocytes. J Immunol 163:2953-2959.

Wiley CA, Schrier RD, Morey M, Achim C, Venable JC, Nelson JA (1991a) Pathogenesis of HIV encephalitis. Acta Pathol Jpn 41:192-196.

Wiley CA, Masliah E, Morey M, Lemere C, DeTeresa R, Grafe M, Hansen L, Terry R (1991b) Neocortical damage during HIV infection. Ann Neurol 29:651-657.

Yeh MW, Kaul M, Zheng J, Nottet HS, Thylin M, Gendelman HE, Lipton SA (2000) Cytokine-stimulated, but not HIV-infected, human monocytederived macrophages produce neurotoxic levels of L-cysteine. J Immunol 164:4265-4270.

Zechner D, Fujita Y, Hulsken J, Muller T, Walther I, Taketo MM, Crenshaw III EB, Birchmeier W, Birchmeier C (2003) Beta-catenin signals regulate cell growth and the balance between progenitor cell expansion and differentiation in the nervous system. Dev Biol 258:406-418.

Zheng J, Thylin MR, Cotter RL, Lopez AL, Ghorpade A, Persidsky Y, Xiong H, Leisman GB, Che M, Gendelman HE (2001) HIV-1 infected and immune competent mononuclear phagocytes induce quantitative alterations in neuronal dendritic arbor: relevance for HIV-1-associated dementia. Neuro Res 3:443-459. 